$\xi=\square$

\title{
Structural and electronic properties of boron- bismuth compound under pressure
}

\author{
Salah Daoud ${ }^{1 *}$, Noudjoud Lebga ${ }^{2,3}$ \\ ${ }^{1}$ Laboratoire Matériaux et Systèmes Electroniques (LMSE), Université Mohamed Elbachir El Ibrahimi de Bordj Bou Arreridj, \\ Bordj Bou Arreridj, 34000, Algérie \\ ${ }^{2}$ Laboratoire d'Optoélectronique et Composants, Faculté des sciences, Université de Sétif 1, Sétif, 19000, Algérie \\ ${ }^{3}$ Département des sciences de la matière, Faculté des sciences et de la technologie, Université Mohamed \\ Elbachir El Ibrahimi de Bordj Bou Arreridj, Bordj Bou Arreridj, 34000, Algérie \\ *Corresponding author_E-mail: salah_daoud07@yahoo.fr
}

\begin{abstract}
In the present work, we report first principles calculations of the pressure effect on the structural and electronic properties of BoronBismuth (BBi) compound in its zincblende phase. The pseudopotential plane wave (PPW) method in the framework of the density functional theory (DFT) within the local density approximation for the exchange-correlation functional, and the Hartwigzen-GoedeckerHutter $(\mathrm{HGH})$ scheme for the pseudopotential were used in the calculation. The unit cell volume, the molecular and crystal densities, the equation of state and also the linear and quadratic pressure coefficients of the energy band-gaps are investigated.
\end{abstract}

Keywords: Density Functional Theory; Structural and Electronic Properties; III-V Materials.

\section{Introduction}

Among the III-V materials, the III-V compounds based on bismuth element have attracted both scientific and technological interest in recent years [1], [2]. Thus adding bismuth to III-V semiconductors is of great importance for numerous optical and electronic applications, especially in the fabrication of the solar cells, and also in elaboration of the transistors and devices based on spintronics [1].

The density-functional perturbation theory (DFPT) was widely applied to predict the phonon spectra, interatomic force constants, born effective charges and a variety of other properties of solid state materials. This method was used by Bouamama et al. [2] to investigate the lattice dynamics and dielectric properties of $\mathrm{BB}$ material in its zincblende structure.

The effect of hydrostatic pressure on the electronic band-gaps is also studied by Bouamama et al. [2]; they found that the BBi material is a semiconductor with indirect band-gap. In fact, they mentioned that the obtained values of the indirect band-gap energy decrease with increasing of hydrostatic pressure.

Using the full potential linearized augmented plane wave method, and as implemented in the WIEN2k code, the electronic behavior of BBi material was also studied by Madouri et al [3]. They found that this material is a semiconductor with direct band-gap with a small optical transition of about $0.13 \mathrm{eV}$.

Under application of external stress (hydrostatic pressure for example) the zincblende phase is destabilized and structural phase transition occurs. For (B3) BBi compound, there are very little results [4-9] on the pressure induced phase transition.

In the present work, we report first principles calculations of the hydrostatic pressure effect on the unit cell volume, molecular and crystal densities, the equation of state and finally the linear and quadratic pressure coefficients of the energy band gaps using the pseudopotential plane wave method, in the framework of the density functional theory (DFT) within the local density approximation (LDA).

\section{Computational methods}

The first-principle calculations were performed by employing pseudopotential plane-waves approach based on the density functional theory [10] and implemented in the ABINIT code [11]. ABINIT code is a common project of the Universite Catholique de Louvain, Corning Incorporated and other contributors. The Teter and Pade parameterization [12] for LDA was applied for the exchange-correlation functional. The effect of the inner electrons and the nucleus (the frozen core) was described within a pseudopotential scheme; the Hartwigzen-Goedecker-Hutter (HGH) scheme [13] to generate the pseudopotentials was used. A planewave basis set was used to solve the Kohn-Sham equations in the pseudopotential implementation of the DFT-LDA.

\section{Results and discussions}

\subsection{Convergence study}

Before starting the calculation of structural and electronic properties desired, it is necessary to know the optimal values of the plane wave kinetic energy cut-off (Ecut), and the grid of special $k$ points (nkpt). So several sets of convergence tests have been carried out in order to choose correctly those two important parameters.

In Fig. 1, we plot the variation of total energy versus Ecut and versus nkpt. The chosen plane wave kinetic energy cut-off is 90 Hartree for (B3) BBi compound. For the (B3) BBi material, we adopt $(4 \times 4 \times 4)$ shifted Monkhorst-Pack k-point. 

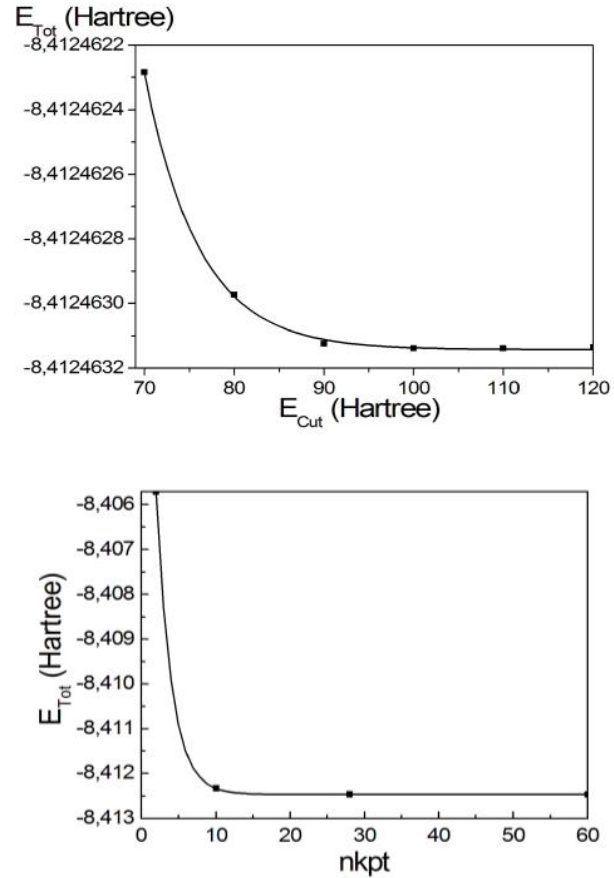

Fig. 1: Total Energy versus $E_{\text {cut }}$ and Versus nkpt in (B3) BBi Material.

\subsection{Structural properties}

\subsubsection{Geometry optimization}

After obtaining Ecut and the nkpt which give the best convergence possible of the total energy. These two parameters are used in the calculation of the total energy for various values of the lattice constant. The values obtained of the total energy are traced as a function of the unit cell volume. One can deduce the static structural properties such as the equilibrium lattice constant from the volume which gives the minimum energy, the bulk modulus and its pressure derivatives by using the Murnaghan Equation of State (EOS) [14], [15]

$$
E(V)-E\left(V_{0}\right)=\frac{B_{0} V}{B_{0}^{\prime}}\left[\frac{\left(V_{0} / V\right)^{B_{0}^{\prime}}}{B_{0}^{\prime}-1}+1\right]-\frac{B_{0} V_{0}}{B_{0}^{\prime}-1}
$$

Where: $\mathrm{B}_{0}$ is the bulk modulus at zero pressure, $\mathrm{V}_{0}$ is the equilibrium volume, $\mathrm{E}\left(\mathrm{V}_{0}\right)$ is the energy corresponding to equilibrium volume, $B_{0}^{\prime}$ is the pressure derivative of the bulk modulus at $\mathrm{P}=0$. The bulk modulus $\mathrm{B}_{0}$ is given by the following formula:

$$
B_{0}=V\left(\partial^{2} E / \partial V^{2}\right)=(4 / 9 a)\left(\partial^{2} E / \partial a^{2}\right)
$$

Where a is the lattice constant.

The Murnaghan Equation of State (EOS) originates from the observation that the pressure derivative of the bulk modulus shows negligible pressure dependence [15]. Therefore, we can make the following approximation [15].

$$
\frac{\partial B}{\partial P} \approx B_{0}^{\prime}=\left.\frac{\partial B}{\partial P}\right|_{V=V_{0}}=-\frac{V}{B} \frac{\partial B}{\partial V}
$$

Fig. 2 shows the variation of the total energy as a function of the volume of one molecule of both zincblende (B3) and Rock-salt (B1) phases of BBi compound. From the graphs connecting the total energy and relative volume one can clearly see that the (B3) phase is more stable than the (B1) phase.
The values of the lattice parameter $\mathrm{a}_{0}$, bulk modulus $\mathrm{B}_{0}$, and its pressure derivative $\mathrm{B}_{0}{ }^{\prime}$ of both zincblende and Rock-salt phases are presented in table.1, and compared with the available theoretical data [1], [2], [3], [5], [6], [8], [16], [17].
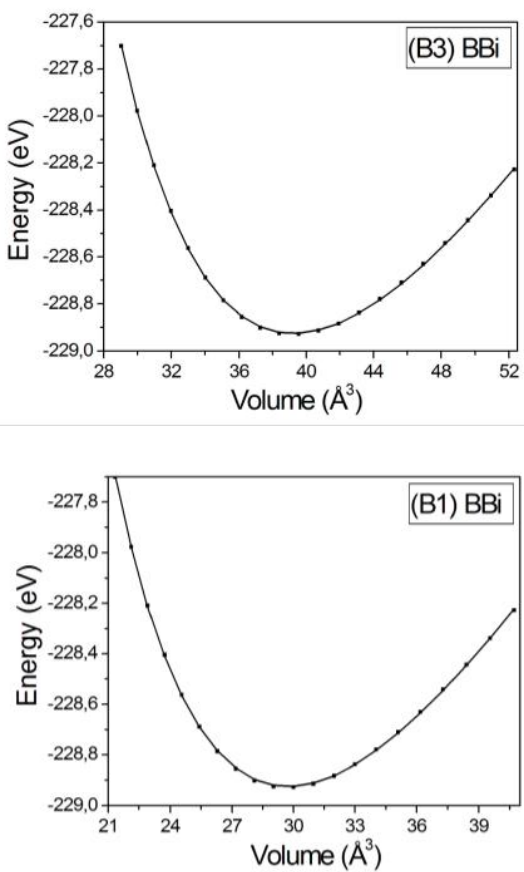

Fig. 2: Total Energy versus $E_{\text {cut }}$ and Versus Volume in both Zincblende and Rock-Salt Phases of BBi Material.

It is seen that, our calculated lattice parameter $\mathrm{a}_{0}$ of both zincblende and Rock-salt phases are in general in good agreement with the other theoretical data [1], [2], [3], [5], [6], [8], [16], and [17]. Where for example, the value of lattice constant obtained by us in the case of zincblende phase deviates from the theoretical values [6], [17] within only $0.39 \%$ and $0.07 \%$ respectively.

Our calculated values of bulk modulus $\mathrm{B}_{0}$ and its pressure derivative $\mathrm{B}_{0}{ }^{\prime}$ of both zincblende and Rock-salt phases are also in general in good agreement with the other theoretical data [1], [3], [5], [6], [8], [16], and [17]. Where for example, the value of bulk modulus $\mathrm{B}_{0}$ obtained in this work in the case of Rock-salt phase deviates from the theoretical value of Cui et al. [5] within only $0.60 \%$.

Table 1: Lattice Constants $\mathrm{a}_{0}$, Bulk Modulus $\mathrm{B}_{0}$ and Its Pressure Deriva-

\begin{tabular}{|c|c|c|c|}
\hline Phase & $\begin{array}{l}\text { Results } \\
\text { Parameter }\end{array}$ & $\begin{array}{l}\text { Our } \\
\text { work }\end{array}$ & Other works \\
\hline \multirow{5}{*}{ (B3) } & & & $\begin{array}{llll}5.371[1] & 5.392[2] & 5.529[3] & 5.464[5]\end{array}$ \\
\hline & $\mathrm{a}_{0}(\AA)$ & 5.394 & $5.415[6] 5.448[8] 5.535[16] 5.390[17]$ \\
\hline & $\mathrm{B}_{0}(\mathrm{GPa})$ & 83.99 & $\begin{array}{lllll}87.3[1] & 72.209 & {[3]} & 81.89[5] & 99.30[6] \\
85.87[8] & 72.30[16] & 87.7[17]\end{array}$ \\
\hline & $\mathrm{B}_{0}^{\prime}$ & 4.36 & $4.73[3] 4.32[5] 4.51[6] 2.903[8] 4.52[17]$ \\
\hline & $\mathrm{a}_{0}(\AA)$ & 4.918 & $5.198[5] 5.22[6] 5.234[8]$ \\
\hline \multirow[t]{2}{*}{ (B1) } & $\mathrm{B}_{0}(\mathrm{GPa})$ & 91.9 & $91.35[5] 112.55[6] 111.68[8]$ \\
\hline & $\mathrm{B}_{0}^{\prime}$ & 4.05 & $4.54[5] 3.83[6] 3.21[8]$ \\
\hline
\end{tabular}
tive $\mathrm{B}_{0}{ }^{\prime}$ of $\mathrm{BBi}$ Material In Comparison with Available Theoretical Values [1], [2], [3], [5], [6], [8], [16], and [17].

\subsubsection{Variation of the volume under pressure}

In order to show how the structural parameters behave under hydrostatic pressure, the equilibrium geometries of (B3) BBi unit cells were computed at fixed values of applied hydrostatic pres- 
sure in the 0 to $25 \mathrm{GPa}$ range, where at each pressure value, a complete optimization for the volume unit cells was performed.

In Fig. 3, we plot the variation of the unit cell volume and the relative unit cell volume $\left(\Delta \mathrm{V} / \mathrm{V}_{0}\right)$ as function of applied hydrostatic pressure.

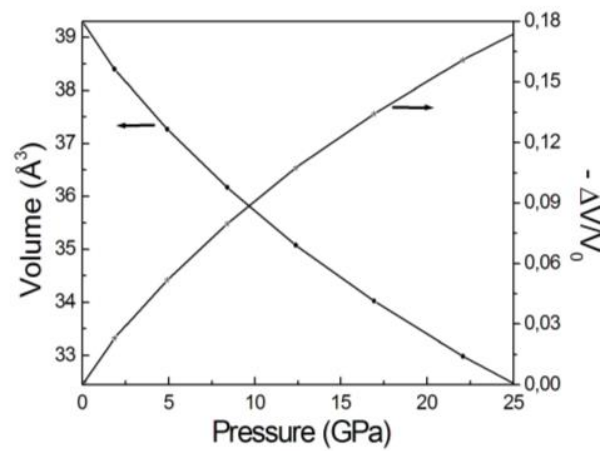

Fig. 3: Variations of the Volume and $\left(\Delta V / V_{0}\right)$ As Function of Pressure for (B3) BBi Material.

\subsubsection{Molecular and crystal densities}

The structure and composition of crystals can be used to calculate their crystal densities. The density $\mathrm{g}$ is given by the following formulas $[18,19]$

$$
g=\frac{Z \cdot M \cdot u}{a \cdot b \cdot c \sqrt{\sin ^{2} \alpha+\sin ^{2} \beta+\sin ^{2} \delta-2(1-\cos \alpha \cdot \cos \beta \cdot \cos \delta)}}
$$

Where: $\mathrm{Z}$ is the number of formula units in a crystal unit cell, $\mathrm{M}$ is the molecular weight of a formula unit in amu (for $\mathrm{BBi}, \mathrm{M}=$ 219.791uma), $u$ is weight of an amu, a, b and c are unit cell axes lengths, and $\alpha, \beta$ and $\delta$ are unit cell axes angles.

For the cubic zincblende-type semiconductors, the molecular density is given by the following expression [20]

$\mathrm{d}_{\mathrm{M}}=4 / \mathrm{a}^{3}$

Where: $\mathrm{a}$ is the lattice parameter.

The calculated molecular and crystal densities at different values of pressure, for (B3) BBi are plotted in Fig. 4.

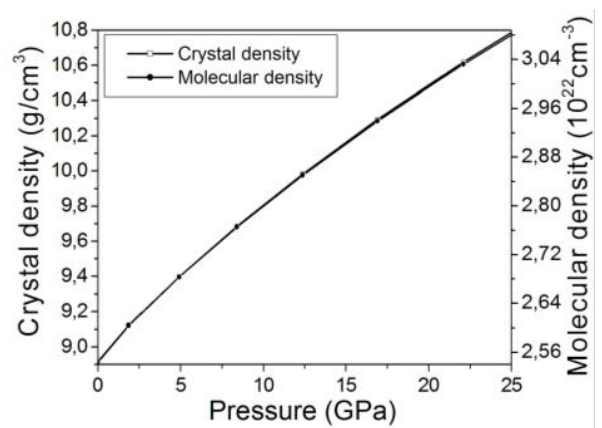

Fig. 4: Variations of the Molecular and Crystal Densities with Pressure of (B3) BBi Material.

Analytical relations for the pressure dependence of molecular and crystal densities are given by the following a quadratic fits:

$\mathrm{d}_{\mathrm{M}}\left(10^{22} \mathrm{~cm}^{-3}\right)=2.551+0.027 \mathrm{p}-2.351 \times 10^{-4} \mathrm{p}^{2} ;$

$\mathrm{g}=8.935+0.095 \mathrm{p}-8.233 \times 10^{-4} \mathrm{p}^{2}$.

The value of the density $g$ of (B3) BBi material at equilibrium is equal to: $8.935 \mathrm{~g} / \mathrm{cm}^{3}$, this value is in good agreement with the theoretical value $\left(8.676 \mathrm{~g} / \mathrm{cm}^{3}\right)$ obtained by Ustundag et al. [21]; the deviation is less than $3 \%$.

\subsubsection{Equation of state}

The understanding of equation of state (EOS) is of primary interest in both the experimental and theoretical high pressure phase transition investigations [22]. The study of equation of state (EOS) helps to understand the nature of solid state theories and determines the values of fundamental thermodynamic parameters [22]. So for studying P-V relation we have used the Vinet equation of state. The Vinet expression $[23,24]$ for the equation of state (EOS) is written as follow [22-24]

$\operatorname{Ln} \mathrm{H}=\operatorname{Ln} \mathrm{B}_{\boldsymbol{0}}+1.5\left(\mathrm{~B}_{\boldsymbol{0}}^{\prime}-1\right)(1-\mathrm{X})$

Where: $\mathrm{X}=\left(\mathrm{V}_{\mathrm{n}}\right)^{1 / 3}$, and $\mathrm{V}_{\mathrm{n}}=\mathrm{V}_{\mathrm{p}} / \mathrm{V}_{0}$ is the normalized volume; where: $V_{p}$ is the volume at a value of pressure no null, and $V_{0}$ is the volume at zero-pressure.

The function $\mathrm{H}$ is given as follow [22-24]

$$
\mathrm{H}=\mathrm{PX}^{2} / 3(1-\mathrm{X})
$$

The universal equation of state (EOS) curve of BBi material in its zincblende phase was depicted in Fig. 5.

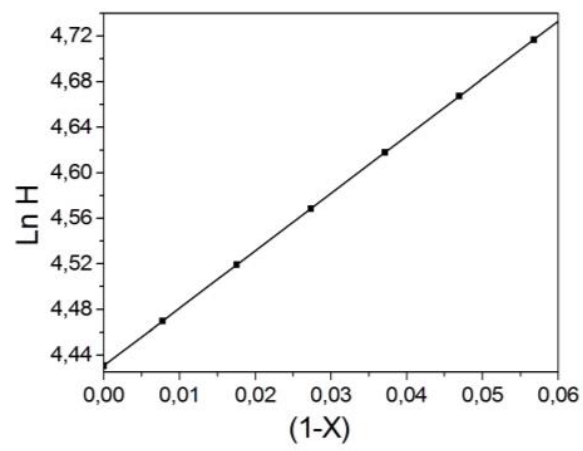

Fig. 5: Universal Equation of State (EOS) of (B3) BBi Material.

Unfortunately as far as we know, there is no data available in the literature on the universal equation of state (EOS) curve for this material. Thus, further theoretical and experimental investigations are significantly needed to confirm the validity of our results.

\subsection{Electronic properties}

\subsubsection{Energy band-gaps-pressure dependence}

Based on the results of the optimized geometry, the electronic band structures of BBi material in its zincblende phase were calculated. The valence band maximum and conduction band minimum occur at different points of the first Brillouin zone, which indicates B3 structure of BBi has indirect gap with about $0.73 \mathrm{eV}$. This result obtained by us is localized between the value $0.61 \mathrm{eV}$ obtained by Cui et al [5], and that obtained by Yalcin [25]. This later mentioned that this material is semiconductor with direct gap of about $0.817 \mathrm{eV}$. This later value was obtained by using the modified Becke-Johnson $(\mathrm{mBJ})$ potential, which is different from the pseudopotentiel used in our work.

In order to investigate the effects of the hydrostatic pressure on the width of the energy band-gaps and position of the conduction band minimum of $\mathrm{BBi}$ compound, the energy band-gaps at selected symmetry points are examined as a function of the hydrostatic pressure increasing. The results of our calculation for the direct and indirect energy band-gaps along the high symmetry directions in the first Brillouin zone for (B3) BBi material versus the hydrostatic pressure up to $25 \mathrm{GPa}$ are shown in Fig. 6.

It can be seen from this figure that the energy band-gaps $E_{\Gamma-\Gamma}$ and $E_{\Gamma-L}$ increase significantly with increasing of pressure, whereas $E_{\Gamma-X}$ and Eg decrease slowly with increasing of pressure. 


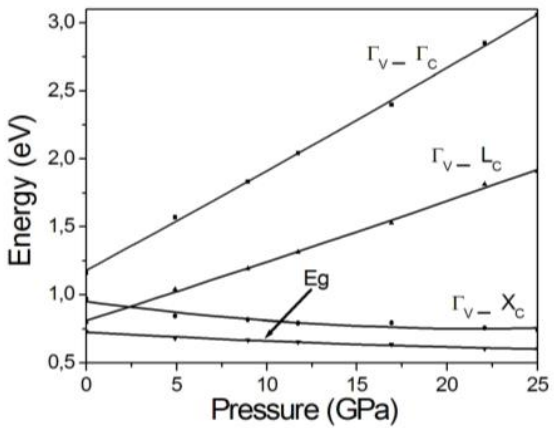

Fig. 6: Variations of the Energy Band-Gaps $E_{\Gamma-\Gamma}, E_{\Gamma-X}, E_{\Gamma-L}$ and $\mathrm{Eg}$ with Pressure of (B3) BBi Material.

For most semiconductors, the variation of the energy band-gaps under pressure can be formulated by the following quadratic expression [26]

$$
\mathrm{E}_{\mathrm{i}}(\mathrm{P})=\mathrm{E}_{\mathrm{i}}(0)+\mathrm{C}_{1} \mathrm{P}+\mathrm{C}_{2} \mathrm{P}^{2}
$$

Where: $c_{1}$ and $c_{2}$ are the linear and quadratic pressure coefficients respectively, and are expressed as: $\mathrm{C}_{1}=\partial \mathrm{E}_{\mathrm{i}} / \partial \mathrm{p}$ in $\mathrm{eV} / \mathrm{Mbar}$, and $\mathrm{C}_{2}$ $=\partial^{2} \mathrm{E}_{\mathrm{i}} / \partial \mathrm{p}^{2}$ in $\mathrm{eV} / \mathrm{Mbar}^{2}$.

The values of the linear and quadratic pressure coefficients as obtained by us are detailed in Table. 2 .

Table 2: Linear and Quadratic Pressure Coefficients Of (B3) BBi Compound.

\begin{tabular}{lll}
\hline \multirow{2}{*}{ Band-gap } & \multicolumn{2}{l}{ Linear and quadratic pressure coefficients } \\
$\mathrm{C}_{1}$ & $\mathrm{C}_{2}$ \\
\hline$\Gamma_{V} \rightarrow \Gamma_{C}$ & 0.072 & $0.13 \times 10^{-3}$ \\
$\Gamma_{V} \rightarrow X_{C}$ & -0.018 & $0.42 \times 10^{-3}$ \\
$\Gamma_{V} \rightarrow L_{C}$ & 0.043 & $0.09 \times 10^{-3}$ \\
$\Gamma_{V} \rightarrow \Delta_{\text {min }}$ & -0.007 & $0.1 \times 10^{-3}$ \\
\hline
\end{tabular}

The behavior of the energy band-gaps of $\mathrm{BBi}$ material in its zincblende phase under hydrostatic pressure is indicated in Fig. 6 , this behavior is very similar to that observed in the case of BSb semiconductor [26], where the fundamental gap remains indirect $\left(\Gamma_{V} \rightarrow \Delta_{\text {min }}\right.$ ). In typical Boron-V (BP, BAs and BSb) materials, the fundamental gap decreases when the pressure increases (volume is compressed) [27].

Unfortunately as far as we know, there is no data available in the literature on the linear and quadratic pressure coefficients for (B3) BBi compound.

Another manner to write the behavior of the energy band-gaps under compression is as a function of the relative variation in lattice constant $\left(-\Delta a / a_{0}\right)$ [27]

Fig. 7 shows the variation of the calculated energy band-gaps as a function the relative variation in lattice constant $\left(-\Delta a / a_{0}\right)$ for $\mathrm{BBi}$ compound in its zincblende phase. It is seen that the bandgaps $\operatorname{Eg}\left(\Gamma_{V} \rightarrow \Delta_{\min }\right)$ and $\Gamma_{V}-X_{C}$ decrease with increase in the relative change of the lattice constant for this compound with negative sign for $\mathrm{A}$ coefficient. The band gaps $\Gamma_{V}-L_{C}$ and $\Gamma_{V}-\Gamma_{C}$ increase significantly with the increasing in the relative variation of the lattice constant $\left(-\Delta a / a_{0}\right)$. This behavior confirms well our analyses on the variation of the band-gaps according to the hydrostatic pressure.

The dependence of the energy band-gaps and $\left(-\Delta a / a_{0}\right)$ can be given through the use of a second-order polynomial fit, this later can be given as follow [27]

$$
E_{i}(a)=E_{i}\left(a_{0}\right)+A\left(-\Delta a / a_{0}\right)+B\left(-\Delta a / a_{0}\right)^{2}
$$

Where: $\Delta \mathrm{a}$ is the change in the lattice constant, $\mathrm{a}_{0}$ the equilibrium lattice paramaer and $\mathrm{i}$ is either the indirect $\Gamma_{V} \rightarrow X_{C}, \Gamma_{V} \rightarrow L_{C}$, Eg and direct $\Gamma_{V} \rightarrow \Gamma_{C}$ band gap.

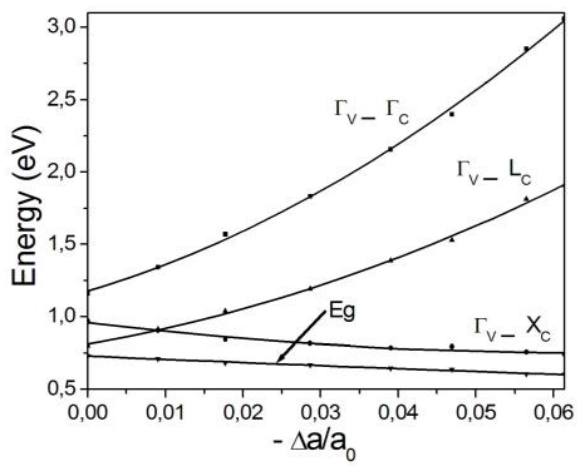

Fig. 7: Relative Lattice Constant Dependence of the Energy Band Gaps: $\mathrm{Eg}, \Gamma_{V}-X_{C}, \Gamma_{V}-L_{C}$ And $\Gamma_{V}-\Gamma_{C}$ of (B3) BBi Material.

The values of the coefficients obtained from this quadratic fit are tabulated in Table 3.

However, as far as we know, there is still no experimental as well as theoretical data of the linear and quadratic coefficients for Boron-Bismuth material. Hence, the present results could be served as a prediction for future experiment.

Table 3: Coefficients Obtained from Least-Square Fits with to the Calculated Values of the Indirect $\Gamma_{V} \rightarrow X_{C}, \Gamma_{V} \rightarrow L_{C}, \Gamma_{V} \rightarrow \Delta_{\min }$ and the Direct $\Gamma_{V} \rightarrow \Gamma_{C}$ Band Gaps of (B3) BBi Material.

\begin{tabular}{llll}
\hline \multirow{2}{*}{ Band-gap } & \multicolumn{3}{l}{$\mathrm{E}_{\mathrm{i}}\left(\mathrm{a}_{0}\right)$, and linear and quadratic pressure coefficients } \\
\hline$\Gamma_{V} \rightarrow \Gamma_{C}$ & 1.18 & 16.03 & $\mathrm{~B}$ \\
\hline$\Gamma_{V} \rightarrow X_{C}$ & 0.96 & -6.58 & 52.35 .19 \\
$\Gamma_{V} \rightarrow L_{C}$ & 0.81 & 9.26 & 141.68 \\
$\Gamma_{V} \rightarrow \Delta_{\text {min }}$ & 0.73 & -2.28 & 3.12 \\
\hline
\end{tabular}

Fig. 8 shows the calculated energy band structure along the high symmetry directions in the first Brillouin zone of BBi material in its zincblende phase at equilibrium (zero-pressure), and at pressure of $22 \mathrm{GPa}$.

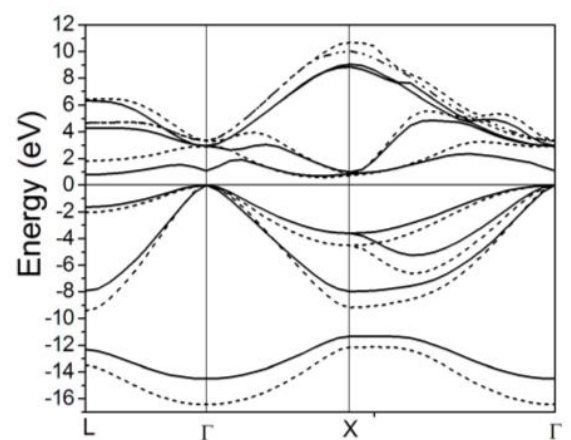

Fig. 8: Calculated Band Structures of BBi Material in Its Zincblende Structure (Solid Line at $\mathrm{P}=0 \mathrm{GPa}$, Dashed with Pressure at 22GPa).

From the figure 8 , it can be seen that the valence band maximum $(\mathrm{VBM})$ and the conduction band minimum $(\mathrm{CBM})$ are found at $\Gamma$ point, and at $\Delta_{\min }$ (near $\mathrm{X}$ point) region respectively. It is clear from this figure that the conduction bands are shifted upwards and valance bands are shifted downwards when the hydrostatic pressure is applied (dashed curves) while the profile of the bands re- 
mains in general unchanged under the application of the hydrostatic pressure.

\section{Conclusion}

A summary of the results obtained in this work is given below: At equilibrium, the lattice parameter, the bulk modulus and its pressure derivative of both zincblende and Rock-salt phases of this material are obtained; they are in general in agreement with the other theoretical data of the literature. The crystal density of the zincblende phase is also determined; it is also in good agreement with the other theoretical data of the literature.

At equilibrium, the valence band maximum and the conduction band minimum of the zincblende phase are found at $\Gamma$ point and at $\Delta_{\min }$ (near $\mathrm{X}$ point) region respectively, indicating that the Boron- Bismuth compound is an indirect semiconductor. Our result of energy gap $(0.73 \mathrm{eV})$ is localized between other theoretical results of the literature.

The pressure effect on the unit cell volume, molecular and crystal densities, equation of state (EOS) and also on the size of the energy band-gaps of Boron- Bismuth (BBi) compound in zincblende phase have been also investigated.

The linear and quadratic pressure coefficients have been determined. The behavior of the band gap variation under high pressure is very similar to that found in other $\mathrm{B}-\mathrm{V}$ (BP, BAs, and $\mathrm{BSb})$ materials; where the fundamental gap decreases very slowly with the increasing of hydrostatic pressure.

Unfortunately as far as we know, there is no experimental data available in the literature on the all quantities predicted in this work for this material. Thus, further theoretical and experimental investigations are significantly needed to confirm the validity of our results on different quantities predicted.

\section{References}

[1] A. Belabbes, A. Zaoui, \& M. Ferhat, "Lattice dynamics study of bismuth III - V compounds", Journal of Physics Condensed Matter, Vol. 20, No. 41 (2008), pp. 415221 - 415224. http://iopscience. iop.org/article/10.1088/09538984/20/41/415221/pdf. http://dx.doi.org/10.1088/0953-8984/20/41/415221.

[2] Kh. Bouamama, P. Djemia, N. Lebgaa \& K. Kassali, "Ab initio calculation of the lattice dynamics of the Boron group- $\mathrm{V}$ compounds under high pressure", High Pressure Research, Vol. 27, No. 2 (2007), pp. 269 -277. http://www.tandfonline.com/ doi/abs/10.1080 /08957950701265359\#.VnmY12zLTIU. http://dx.doi.org/10.1080/08957950701265359.

[3] D. Madouri \& M. Ferhat, "How do electronic properties of conventional III-V semiconductors hold for the III-V boron bismuth BBi compound?" Physica Status Solidi (b), Vol. 242, No.14, (2005), pp. 2856 - 2863. http://dx.doi.org/10.1002/pssb.200441121.

[4] S. Daoud, N. Bioud \& N. Lebgaa, " Elastic and piezoelectric properties, sound velocity and Debye temperature of (B3) BBi compound under pressure", Pramana journal of physics, Vol. 81, No. 5 , (2013), pp. 885-892. www.ias.ac.in/pramana/v81/p885/fulltext.pdf.

[5] S. Cui, W. Feng, H. Hu, Z. Feng \& Y. Wang, "First principles studies of phase stability, electronic and elastic properties in BBi compound", Computational Materials Science, Vol.47, No.4, (2010), pp. 968-972. http://www.sciencedirect.com/science/article/pii/S092702 5609004418

[6] E. Deligöz, K. Colakoglu, Y. O. Ciftci, \& H. Ozisik, "The firs principles study on boron bismuth compound", Computational Materials Science, Vol.39, No.3, (2007), pp. 533-540. http://www. sciencedirect.com/science/article/pii/S0927025606002321 http://dx.doi.org/10.1016/j.commatsci.2006.08.004.

[7] S. Singh, \& M. Sarwan, "Structural phase transition and high pressure elastic behavior of BX $(\mathrm{X}=\mathrm{Sb}, \mathrm{Bi})$ compounds", Journal of optoelectronics and advanced materials, Vol.12, No.10, (2010), pp. 2106-2112. http://www.joam.inoe.ro/download.php?idu=2623.

[8] K. Amara, B. Soudini, D. Rached \& A. Boudali, "Molecular dynamics study on thermomechanical properties of cubic BBi", Computational Materials Science, Vol. 44, No. 2, (2008) pp. 635-640. http://dx.doi.org/10.1016/j.commatsci.2008.04.023.

[9] S. Daoud, "Mechanical properties of BBi compound under pressure ", International Journal of Scientific World: Vol. 3, No. 1 (2015), pp. 69 -75. http://www.sciencepubco.com/index.php/IJSW/article /view/4218. http://dx.doi.org/10.14419/ijsw.v3i1.4218.

[10] E. Engel \& R. M Dreizler, "Density Functional Theory", SpringerVerlag, New York, (2011). http://dx.doi.org/10.1007/978-3-642$14090-7$.

[11] X. Gonze, G. M. Rignanese, M. Verstraete, et al. "A brief introduction to the ABINIT software package", Zeitschrift für Kristallographie, Vol.220, No.5-6, (2005), pp. 558-562. http://dx.doi.org/10.1524/zkri.220.5.558.65066.

[12] S. Goedecker, M. Teter \& J. Hutter, "Separable dual-space gaussian pseudopotentials", Physical Review B, Vol.54, No.3, (1996), pp. 1703-1710. http://dx.doi.org/10.1103/PhysRevB.54.1703.

[13] C. Hartwigsen, S. Goedecker \& J. Hutter, "Relativistic separable dual-space gaussian pseudopotentials from $\mathrm{H}$ to $\mathrm{Rn}$ ", Physical Review B, Vol.58, No.7, (1998), pp. 3641-3662. http://dx.doi.org/10.1103/PhysRevB.58.3641.

[14] F. Murnaghan, "The Compressibility of Media under Extreme Pressures", Proceedings of the National Academy of Sciences of the United States of America, Vol. 30, No. 9, (1944), pp. 244-247. http://dx.doi.org/10.1073/pnas.30.9.244.

[15] L. Vitos, "Computational quantum mechanics for materials engineers", Springer-Verlag London Limited, (2007). ISBN 978184628-950-7. http://link.springer.com/book/10.1007\%2F978-184628-951-4

[16] A. Zaoui, D. Madouri \& M. Ferhat, "First-principles study of the ground state stability of III-V bismuth compounds", Philosophical Magazine Letters, Vol.89, No.12, (2009), pp.807-813, http://www.tandfonline.com/doi/abs/10.1080/09500830903304125. http://dx.doi.org/10.1080/09500830903304125.

[17] S. Q. Wang \& H. Q. Yet, "Plane-wave pseudopotential study on mechanical and electronic properties for IV and III-V crystalline phases with zinc-blende structure", Physical Review B, Vol.66, (2002), p 235111 (7pp). link.aps.org/doi/10.1103/PhysRevB.66.2 35111.

[18] W. J. Tropf, M. F. Thomas \& T. J. Harris, "Properties of crystals and glasses", Handbook of Optics, Vol. IV, McGraw-Hill, New York, (2004). http://photonics.intec.ugent.be/education/IVPV/res_ handbook/v2ch33.pdf.

[19] S. Daoud \& N. Bioud "Structural properties of (B3) TIP under pressure", International Journal of Physical Research, Vol. 2, No. 2, (2014), pp. 50-55. http://www.sciencepubco.com/index.php/IJPR/ article/view/3100. http://dx.doi.org/10.14419/ijpr.v2i2.3100.

[20] S. Adachi, "Properties of Group-IV, III-V and II-VI Semiconductors", John Wiley \& Sons, England, (2005). ISBN 0-470-09032-4. http://dx.doi.org/10.1002/0470090340.

[21] M. Ustundag, M. Aslan, \& Battal G. Yalcin, "The first-principles study on physical properties and phase stability of Boron-V (BN, $\mathrm{BP}, \mathrm{BAs}, \mathrm{BSb}$ and $\mathrm{BBi}$ ) compounds", Computational Materials Science, Vol. 81, (2014) pp. 471-477.

[22] P. Bhardwaj, M. Sarwan, R. Dubey, S. Singh, "Structural and mechanical properties of actinide pnictides", Journal of Molecular Structure, Vol. 1043, No. 1, (2013), pp. 85-90. http://www.science direct.com/ science/journal/00222860/1043

[23] P. Vinet, J. Ferrante, J. R. Smith, J. H. Rose, "Temperature effects on the universal equation of state of solids", Physical Review B, Vol.35, (1987), pp. 1945-1953. http://journals.aps.org/prb/abstract/10.1103/PhysRevB.35.1945. http://dx.doi.org/10.1103/PhysRevB.35.1945.

[24] P. Vinet, J. Ferrante, J. R. Smith, J. H. Rose, " Universality in the Compressive Behavior of Solids", NASA Technical Memorandum 87303, N 86-26775, (1986). http://ntrs.nasa.gov/archive /nasa/casi. ntrs.nasa. gov /19860019303.pdf.

[25] B. G. Yalcin, "Band gap characterization of ternary $\mathrm{BBi}_{1-\mathrm{x}} \mathrm{N}_{\mathrm{x}}(0 \leq \mathrm{x}$ $\leq 1)$ alloys using modified Becke-Johnson $(\mathrm{mBJ})$ potential", Physica B, Vol. 462, (2015), pp. 64-69. http://www.researchgate. net/publication/271510039. http://dx.doi.org/10.1016/j.physb.2015.01.021.

[26] S. Daoud, N. Bioud, N. Lebgaa, L. Belagraa \& R. Mezouar, "Pressure effect on structural, elastic and electronic properties of (B3) BSb compound", Indian Journal of Physics, Vol. 87, No. 4, (2013), pp. 355-362. http://link.springer.com/article/10.1007\%2Fs 12648012-0231-y.

[27] S. Labidi, H. Meradji, S. Ghemid, S. Meçabih, B. Abbar, " Pressure dependence of electronic and optical properties of zincblende BP, BAs and BSb compounds", Journal of Optoelectronics and Advanced Materials, Vol. 11, No. 7, (2009), pp. 994 - 1001 $\mathrm{http}: / /$ joam.inoe.ro/index.php?option=magazine \&op=view\&idu=19 89 \& catid $=40$. 